\title{
Signal Processing Schemes for Doppler Global Velocimetry
}

\author{
James F. Meyers \\ Joseph W. Lee \\ NASA - Langley Research Center \\ Hampton, Virginia 23665 \\ and
}

Angelo A. Cavone

ViGYAN, Inc.

Hampton, Virginia 23665

IEEE - 14th International Congress on Instrumentation in Aerospace Simulation Facilities Rockville, Maryland October 27-31, 1991 



\title{
Signal Processing Schemes for Doppler Global Velocimetry
}

\author{
by \\ James F. Meyers \\ Joseph W. Lee \\ NASA - Langley Research Center \\ Hampton, Virginia 23665 \\ and \\ Angelo A. Cavone \\ ViGYAN, Inc. \\ Hampton, Virginia 23665
}

\begin{abstract}
Two schemes for processing signals obtained from the Doppler global velocimeter are described. The analog approach is a simple, real time method for obtaining an RS-170 video signal containing the normalized intensity image. Pseudo colors are added using a monochromatic frame grabber producing a standard NTSC video signal that can be monitored and/or recorded. The digital approach is more complicated, but maintains the full resolution of the acquisition cameras with the capabilities to correct the signal image for pixel sensitivity variations and to remove of background light. Prototype circuits for each scheme are described and example results from the investigation of the vortical flow field above a 75-degree delta wing presented.
\end{abstract}

\section{Introduction}

The use of high lift devices to increase the performance envelope of advanced aircraft results in flow fields comprised of vortical and reversed flows with turbulent separations interacting with lift and control surfaces. These complicated flows are difficult to measure and as yet have not been modeled using computational fluid dynamics (CFD). In order to develop code necessary to describe the flow, especially time dependent modeling, instrumentation must be developed to be capable of measuring the flow without influencing it. This requirement disqualifies all probe measurements. Not only are they incapable of measuring reversed or highly turbulent flow, they influence the flow causing vortex movement around the probe or even 
premature vortex bursting.

ptical techniques such as fringe type laser velocimetry will not influence the flow and are capable of measuring the full three-component velocity vector. Fringe type laser velocimeters have been used to measure such complicated flows as helicopter rotor inflows, leading edge vortices from delta wings, and Taylor-G rtler vortices within laminar boundary layers in wind tunnels. These measurements have greatly increased the understanding of these flow phenomena and serve as databases for the development and verification of CFD codes. A disadvantage of this point measurement technique is the length of time necessary to obtain measurements over a detailed grid needed to characterize the flow field. In response to this problem, a new class of laser velocimetry techniques is being developed-Global Velocimetry. The first technique developed in this class was particle image velocimetry. This technique uses a photographic camera to obtain a double exposure of the particle field within the flow using a pulsed laser light sheet. While this technique obtains simultaneous measurement of velocities over the viewing area, it has four drawbacks limited viewing area (typically inch $\$$, limited to two velocity component measurements, directional ambiguity, and long data processing times. Although these drawbacks may be reduced, or in some cases even eliminated with further research, a new global technique-Doppler Global Velocimetry-is being developed which has the advantage of particle image velocimetry without its drawbacks.

\section{The Basic Principle}

In the manner of the original laser velocimeter developed by Yeh and Cummins, reference 1, Doppler global velocimetry (DGV) utilizes the shift in frequency imposed on light scattered from a particle passing through a laser beam by the Doppler effect to determine the velocity of the particle. The propagation direction of the laser beam,

, and the viewing direction, , determine the direction where a motion produces the Doppler shift. This direction is the vectorial difference between

and , that is - As shown in figure 1, the measuremen also be found by determining the vector perpendicular to the bisector of vectors and lying within the plane defined by the vectors Thus for a general flow velocity vector $\mathbf{V}$, the shift in frequency, $\Delta v$, of the collected scattered laser light is dependent on the vectorial dot product between the velocity vector $\mathbf{V}$ and the measurement vector as shown in equation 1

$$
\Delta v=\frac{v_{o}(-) \cdot V}{C}
$$


where $v_{0}$ is the optical frequency of the laser light sheet and $c$ is the speed of light. The dependence on this vectorial relationship allows the measurement of other velocity components by choosing various viewing directions and/or laser propagation directions.

Yeh and Cummins, reference 1, viewed the scattered light from particles passing through the waist of a focused laser beam using the optical arrangement shown in figure 2 . The collected scattered light was placed collinear with a portion of the laser beam and directed to a photomultiplier. The two light waves heterodyne on the photocathode surface yielding an electronic signal containing the difference frequency, $\Delta v$, defined in equation 1 . The Doppler frequency can then be measured using a spectrum analyzer or oscilloscope, or specially designed instrumentation for laser velocimetry applications such as a frequency tracker, reference 2, or high-speed burst counters, references $3-5$. sing the heterodyne technique to obtain the Doppler frequency cannot be extended to global measurements since the allowable acceptance angle to maintain the heterodyne process is too limited, reference 6 .

Direct measurement of the Doppler shift in the scattered light was attempted by Self in $1 \quad 7 \quad$ reference 7 , using interferometer. This approach was necessary since the high velocity in this research induced a Doppler frequency above the frequency response of a photomultiplier. While the interferometer provided acceptable results for this specific application, its limited acceptance angle prohibits extension to global measurements.

Instead of a Fabry-Perot interferometer, Seiler and Sruli es, reference , used a Michelson interferometer in an attempt to directly measure the Doppler frequency. Not only were they successful, they were able to generate global measurements of the flow producing pictures of fringe maps of constant phase shift between the direct and time delayed collected scattered light. These fringes indicate locations where the Doppler shifts in the scattered light are multiples of the phase delay in the interferometer. The difficulty in maintaining alignment of the interferometer and the still relatively narrow acceptance angle limit the application of this technique.

Departing from interferometric approaches, et rali,mee,fer en ce used the absorption properties of molecular Iodine to serve as an optical frequency discriminator to develop the Doppler global velocimeter. An Argon ion laser operating in single-line mode at $51 \quad .5 \mathrm{~nm}$ is tuned (by tilting the intercavity etalon) to an optical frequency corresponding to a point midway along the edge of an absorption line of the Iodine absorption line filter (ALF), figure 3. Collected scattered light from a 
stationary ob it passes through the ALF. If the ob ect or cloud of particles will be attenuated by 50 percent as ect or particle cloud is moving, the attenuation through the ALF will increase (or decrease, depending on the direction of movement) by an amount proportional to the Doppler shift. $y$ using the ALF as a filter for a CCD camera, an en tire laser light sheet can be viewed and the velocity field determined.

In practice, particle size distribution and number density, and the laser light sheet intensity profile are other factors influencing the amount of collected scattered light reaching the viewing camera. If a second camera, without an ALF, views the same scene, its output can be used as a reference to normalize the viewing camera signal, thus minimizing these influences. The arrangement of the cameras and associated optics are shown in figure

ther influences from nonuniform elements and variations in pixel sensitivities in the CCD cameras are removed using pixel-by-pixel calibration ratios.

\section{Signal Processing}

The removal of influences on the collected scattered light from sources other than the ALF requires both the signal and reference cameras to view the same portion of the light sheet on a pixel basis for exactly the same period of time. This necessitates precise optical alignment and timing control of the two cameras. The acquired images must main tain their synchronization as they are transferred to the signal processor for normalization. Signal processing and ad ustment for other influences on the signal image were performed using both analog and digital approaches.

Camera alignment begins by placing a lined target in the measurement plane and viewing both the signal camera and processed signal images. The signal camera and lens are ad The reference camera is then ad usted to obtain the desired image. magnification ad usted, and the directing mirror ad tilt to minimize the line visibility on the target in the processed signal image. Minimizing the visibility of the lines places the cameras in the required alignment. Ideally the lin es should vanish, but manufacturing variations in the construction of the CCD elements prevent perfect optical alignment. lectronic alignment between two cameras is normally obtained by using the internal horizontal and vertical synchronization clocks from one camera to drive the second. However, the second camera will be one frame behind which prohibits simultaneous acquisition of light by corresponding pixels. Therefore, the internal pixel clocks were disabled and an external synchronization circuit used to simultaneously drive both cameras. This optical and 
electronic alignment assures simultaneous interrogation of corresponding pixels in each camera to obtain identical views in time and space of the light scattered from particles passing through the light sheet.

Standard video frame grabbers are capable of acquiring images from more than one camera only through the use of video multiplexers. The requirement of simultaneous image acquisition by two cameras in DGV applications cannot be accomplished using multiplexers, thus the development of custom signal processing hardware is necessary. The approach used by etralneference , was to develop an analog divider circuit capable of normalizing the signal camera output by the reference camera output in real time. Although the circuit details were not presented in reference , the simplicity of this approach and its real time capabilities made it attractive to develop. The standard RS-170 signal from each camera must be stripped of its embedded retrace synchronization pulse to prepare the signal for the analog divider. Further, the reference signal must be tested for minimum amplitude to avoid division by zero. If the reference signal is too low, a full scale voltage is switched to the divider producing a zero level output thus avoiding the condition where zero is divided by zero. The retrace synchronization pulse from the signal camera is then added to the normalized signal to again produce a standard RS-170 video signal. A block diagram of this circuit is shown in figure 5. The normalized video signal is then acquired by a standard frame grabber which adds pseudo colors to more easily visualize the measured velocity. The colored signal is converted to standard NTSC color video for output to a video monitor and recording on a -MATIC video recorder.

Although the analog circuit has the distinct advantages of simplicity and real time capabilities, it has several disadvantages. Circuit elements to provide an invalid measurement indication for pixels receiving too little or too much scattered light will greatly complicate the circuit. These elements must also be specifically ad the propervalues for the attached cameras. Ad usted to provide output signal to account for the addition of background light cannot be made. Ad ustments to the normalization results to account for pixel sensitivity variations in each camera must be made within the controlling computer, thus prohibiting real time operation. The greater signal processing flexibility needed to provide these capabilities can only be obtained using digital circuitry.

A specialized dual frame grabber was designed with the specific purpose of acquiring synchronized images from two cameras. A block diagram of the proposed system is shown in figure 6 . The synchronized image from each camera is digitized by its respective -bit analog-to-digital 
converter (ADC) which is operated at $10 \mathrm{MHz}$ to obtain $512 \times 512$ pixel image mappings. Since the ADCs are bit, there are 256 possible values and 256 or 65,536 possible results from the normalization operation. Therefore, the most time efficient method of dividing the signal image by the reference image is to use a lookup table containing all possible quotients. The signal ADC provides the row address and the reference ADC provides the column address to select the appropriate cell within the lookup table containing the normalized result. There are several other advan tages of using a lookup table to determine the normalization result. Cells representing conditions where the signal is greater than the reference, low signal levels, low and high reference levels can be loaded with full scale values indicating improper measurement conditions. Since CCD cameras integrate incoming light in a linear manner until it is output, constant background illumination can be removed using the following technique. With the flow off (or the particle generator turned off if the light sheet is moved during testing), signal and reference images are acquired and passed without normalization to the computer. ach image ensemble is then averaged to obtain the background illumination level for its respective camera. A new normalization array is calculated with the respective background illumination levels subtracted from the numerator and denominator for each cell prior to division. For example, assume the average background illuminations from the signal and reference cameras are the values 20 and 25 , respectively. The numerators representing the signal level (initially matching the row numbers in the table) are lowered by 20 . Table rows with addresses of 20 or less are loaded with full scale values indicating an improper measurement condition. Likewise, the denominators representing the reference level (initially matching the column numbers in the table) are lowered by 25 . Table columns with addresses of 25 or less are also loaded with full scale values. Placement of full scale values in cells with column addresses of 250 or greater indicates saturation of the reference signal. Full scale values placed in cells where the signal is greater than the reference represents inappropriate conditions. The remaining cells are loaded with values obtained by solving the following equation

$$
\text { Cell Value }=\frac{255(\text { Signal Value }-20)}{\text { Reference Value }-25}
$$

The value of 255 in equation (2) is used to provide the maximum accuracy obtainable from an -bit lookup table.

Ad ustments to the normalized data for the removal of pixel sensitivity variations in each camera can be made within the digital signal processor instead of the controlling computer as required with the analog approach. A second lookup table containing the mapping of the 
pixel sensitivity ratio of the reference camera sensitivity divided by the signal camera sensitivity for each of the $512 \times 512$ camera elements is sequentially addressed using the camera synchronization signal as the clock. The table address is set behind the camera pixel address to account for circuit timing delays as the data passes through the ADCs and the normalization lookup table. Thus, as the data reaches the multiplier from the normalization lookup table, figure 6 , the sensitivity calibration for that pixel pair is simultaneously available. The corrected data can then be converted to an analog NTSC signal with pseudo color for real time monitoring and video storage, and/or transferred in digital form to the controlling computer for storage.

A prototype dual frame grabber was constructed to encompass the elements of the digital system up to the multiplier shown in figure 6 . The system, shown in figure 7, was designed to transfer the lookup table output directly to the controlling computer. It was also equipped with sufficient storage to hold the images from both cameras allowing their transfer to the computer for cross checking of system operation. In this first effort, the data transfer was kept simple by using standard microprocessor controlled I

data acquisition and normalization operated in real time, the transfer of data to the computer required approximately 20 seconds.

\section{Proof-of-Concept Wind Tunnel Experiment}

The Doppler global velocimeter was tested in the

asic Aerodynamic Research Tunnel ( ART) to determine if the technique was applicable to wind tunnel flow field investigations. The vortical velocity field above a 75-degree delta wing was chosen as the test flow since five-hole probe and fringe-type laser velocimeter investigations of the flow had been previously conducted in the tunnel, references 10 and 11 . These measurements will be used as comparative standards to determine the capabilities of the DGV. The model was placed at an angle of attack of 20.5 degrees to obtain a stable vortical flow. The DGV optical system was configured as shown in figure with the laser light sheet placed normal to the surface of the model. The receiver optical system was placed in forward scatter, rotated 53 degrees from the propagation direction of the laser beam in the horizontal plane. A view of the illuminated vortical flow from the camera position is shown in figure This optical configuration measured the velocity along the direction 26.5 degrees from the streamwise direction in the horizontal plane. The flow was seeded using the propylene glycol vaporization/condensation generator normally used for light sheet flow visualization in reference 10. A typical data acquisition consisted of acquiring ten

ART, 
images using the digital dual frame grabber and five minutes of real time data using the analog signal processor.

The ability of the DGV to measure flow velocities can be illustrated by selecting a test condition. The tunnel dynamic pressure was set to 3 andthe model angle of attack ad usted to 20.5 degrees. The laser light sheet was placed perpendicular to the model at the 70 percent chord location. An example of the data obtained from the DGV using the digital circuitry is shown by the single frame presented in figure example of data obtained using the analog approach is shown by the single frame presented in figure 10 . The images have not been spatially rotated to remove the perspective view of the light sheet as shown in figure nor has any attempt been made to smooth the measurement noise or to remove the reflection of the light sheet from the model. Comparative data obtained from three component measurements resolved along the DGV measurement direction using the five-hole probe and laser velocimeter are shown in figures 11 and 12 respectively. The asymmetry of the velocity contours is due to the inclusion of a portion of the vortical rotation, sin 26.5 degrees, within the measurement direction. The data indicate that velocity at the top of the left vortex should match the bottom of the right with the flow next to the wing being slightly higher due to flow compression. The top of the right vortex should likewise match the bottom of the left. This pattern is clearly illustrated in the single frame DGV results, figures and 10. The velocity characteristics of the vortex flow can be seen by viewing the normalized signal amplitude (inversely proportional to velocity) profile measured by the DGV (digital processing, figure ) through the center of the two vortices, figure 13. (Recall that the normalization circuit is programmed to drive the normalized signal amplitude to full scale if the signal amplitude is below the camera threshold or the scattered light saturates the reference camera.) The high frequency oscillations in the profile are due to sensitivity variations in the CCD elements and element spacing differences between the two cameras. Averaging ten frames of data minimizes variations in velocity from turbulence or electronic noise leaving the fixed sen sitivity variations visualized in the profile through the center of the vortices in figure 1 . If the profile is high pass filtered, the variation due to pixel sensitivities can be isolated and used to develop a calibration to remove the variations. Following conversion to velocity of the profile in figure 13 , the resulting calibration was applied yielding the profile in figure 15. Comparing the velocity profile with the data obtained with the five-hole probe and laser velocimeter indicates proper trends near the vortex core. However, the velocities near the outward edges of the vortices do not appear to be reasonable. These trends may result if the laser frequency is placed too near the nonlinear portion of the absorption line of the ALF. 


\section{Summary}

The Doppler global velocimeter has been presented and shown to have the potential to provide global, multicomponent measurements in real time. $\quad$ mphasis has been placed on the development of signal processing schemes to normalize the signal camera image with the reference camera image. The analog approach was described and shown to provide a simple method for real time signal processing. However, its limitations in signal correction necessitated the development of the digital dual frame grabber as the primary signal processing approach. xample data obtained by the DGV of the vortical flow above a 75-degree delta wing have been presented and compared with previous five-hole probe and fringe-type laser velocimeter data.

\section{References}

1. Yeh, Y. and Cummink, okbl i zed Fluid FI ow M easurements with a He-NeLaser Spectrometer, Applied Physics Letters, Vol. No. 10 , pp. 176-17 , May 1

2. Fridman, J. D. innard, LasEr Doppler and Meist Instrumentation for the Measurement of Turbulence in Gas and Fluid Flows, Proceedings of the Technical Program -Electro-Optical Systems Design Conference, ar1 A. $\begin{array}{llll}\text { Inc., c. } 1 & 70, \text { pp. } 12 & -1 & 6 .\end{array}$

3. Iten, P. D. and MasthaserJ.Doppler Vel oci meter Offering High Spatial and Temporal Resolution, Flow-Its Measurement and Control in Science and Industry, Volume ne, Part Two, R. . Wendt, Jr., ed., Instrument Soc. of Ame 1 , pp. 1007-1013.

Lennert, A. Smith, F. H., Jr. Applicationd of Dual Scatter, Laser, Doppler Velocimeters for Wind Tunnel Measurements, International Congress on Instrumentation in Aerospace Simulation Facilities-

71-C-33 A S, Inst. of

ICII REEord, I

c. 1 71, pp. 157-167.

lectrical and

lectronic

5. Asher, J. A. LDV Systems Development and Testing, The Use of the Laser Doppler Velocimeter for Flow Measurements, W.H. Stevenson and H.D. Thompson, eds., Contract N0001 
6. Huffaker, R. M. Fuller, C. Appl åatilomworence, T. R. L aser Doppl er Vel ocity I nstrumentation to the Measurement of J et Turbulence, International Automotive ngineering Congress, Society of Automotive ngineers, Detroit, MI, January 13-17, 1 6

7. Self, S. A. Laser Doppler Anemometer for Boundary Layer Measurements in High Vel ocity, High Temperature MHD Channel Flows, Proceedings of the Second International Workshop on Laser Velocimetry, Purdue niversity, Vol. II, pp. March 27-2 , 1 7

Seiler, F. and SruDoppler-Pi etsu fles of Vel oci ty Fi el ds, An Application to Fluid Mechanics, Proceedings of the Third International Symposium on Applications of Laser Anemometry to Fluid Mechanics, Lisbon, Portugal, paper 1 .1, July 7omine, $\mathrm{H}$. rosnan, S. J.

Real-Time, Doppler Global Velocimetry, AIAA 2 th Aerospace Sciences Meeting, Reno, NV, paper AIAA- 1-0337, January 7-10, 1 1.

10. Sellers, W. L., II I and The B asic Aleradyn Ami CS Research Tunnel - A Facility Dedicated to Code Validation, AIAA 15th Aerodynamic Testing Conference, San Diego, CA, paper AIAA$-1$

7, May 1 $-20,1$

11. Meyers, J. F. and Hepne⿻l Tasurement of L eading Edge Vortices from a Delta Wing Using a Three Component Laser Velocimeter, AIAA 15th Aerodynamic Testing Conference, San Diego, CA, paper AIAA$-202$

, May 1 $-20,1$ 
Laser Propagation Direction, î

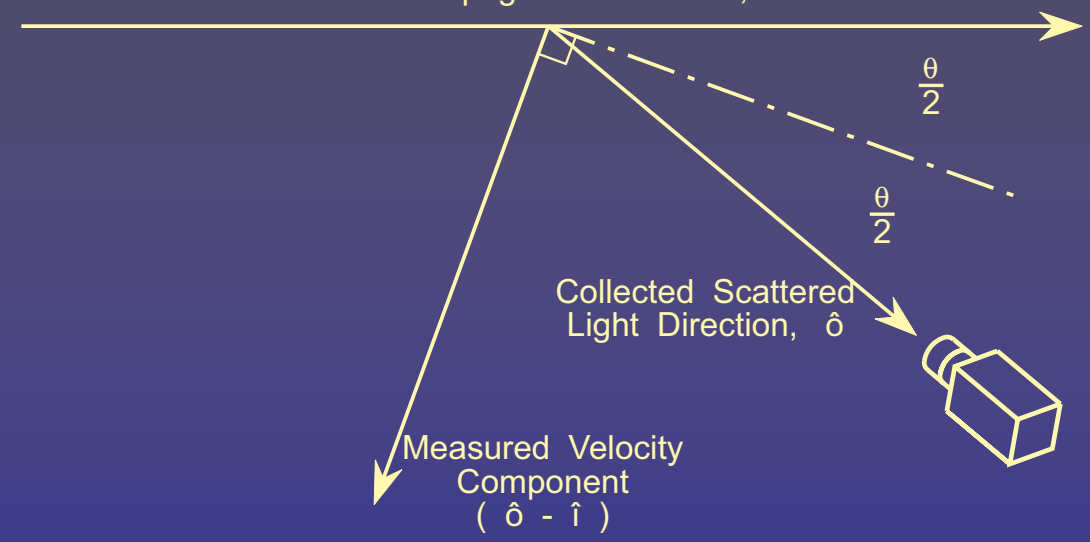

Figure 1.- Determination of measured velocity component direction based on light sheet propagation and receiver location.

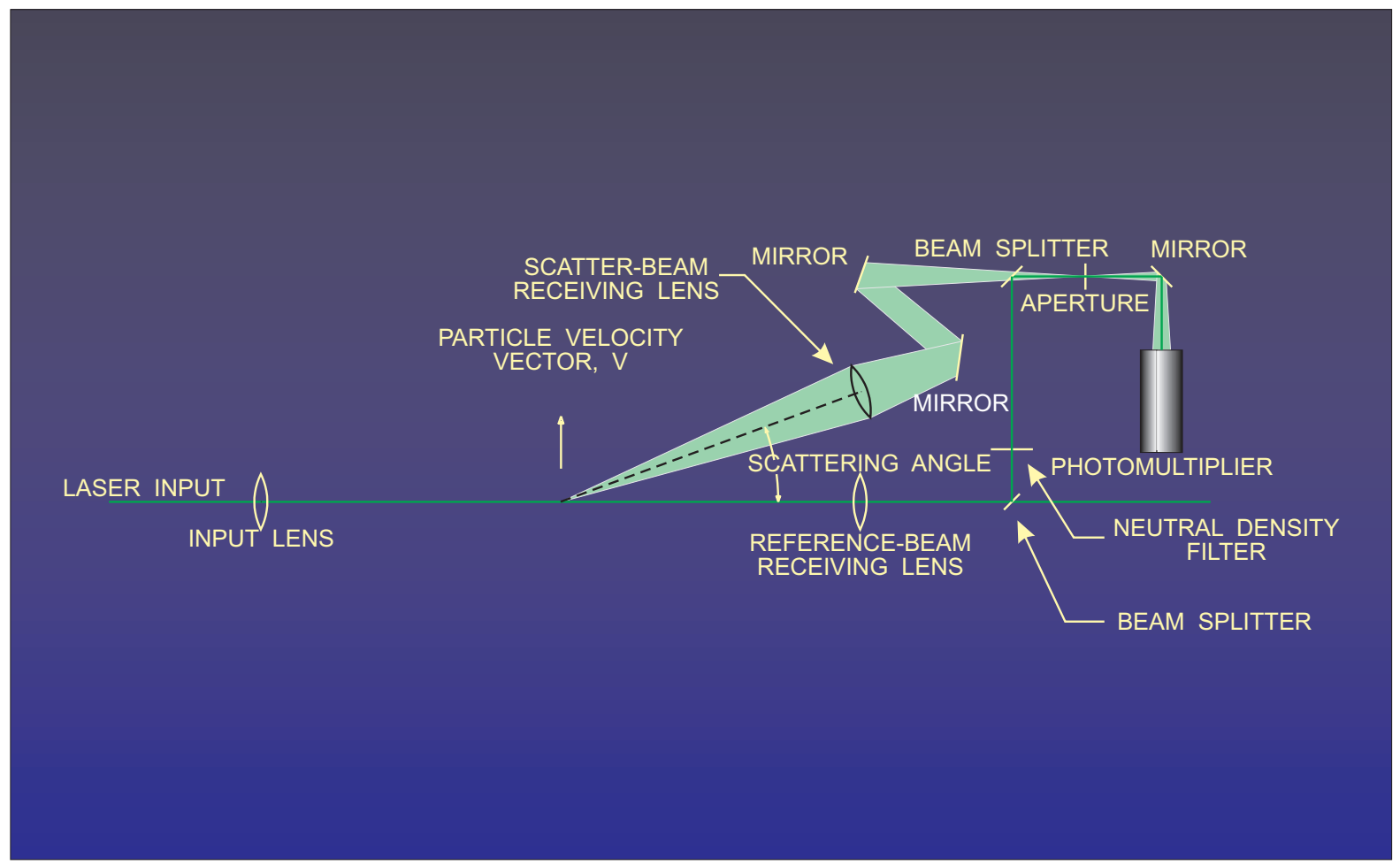

Figure 2.- Diagram of a forward-scatter, reference beam laser velocimeter. 


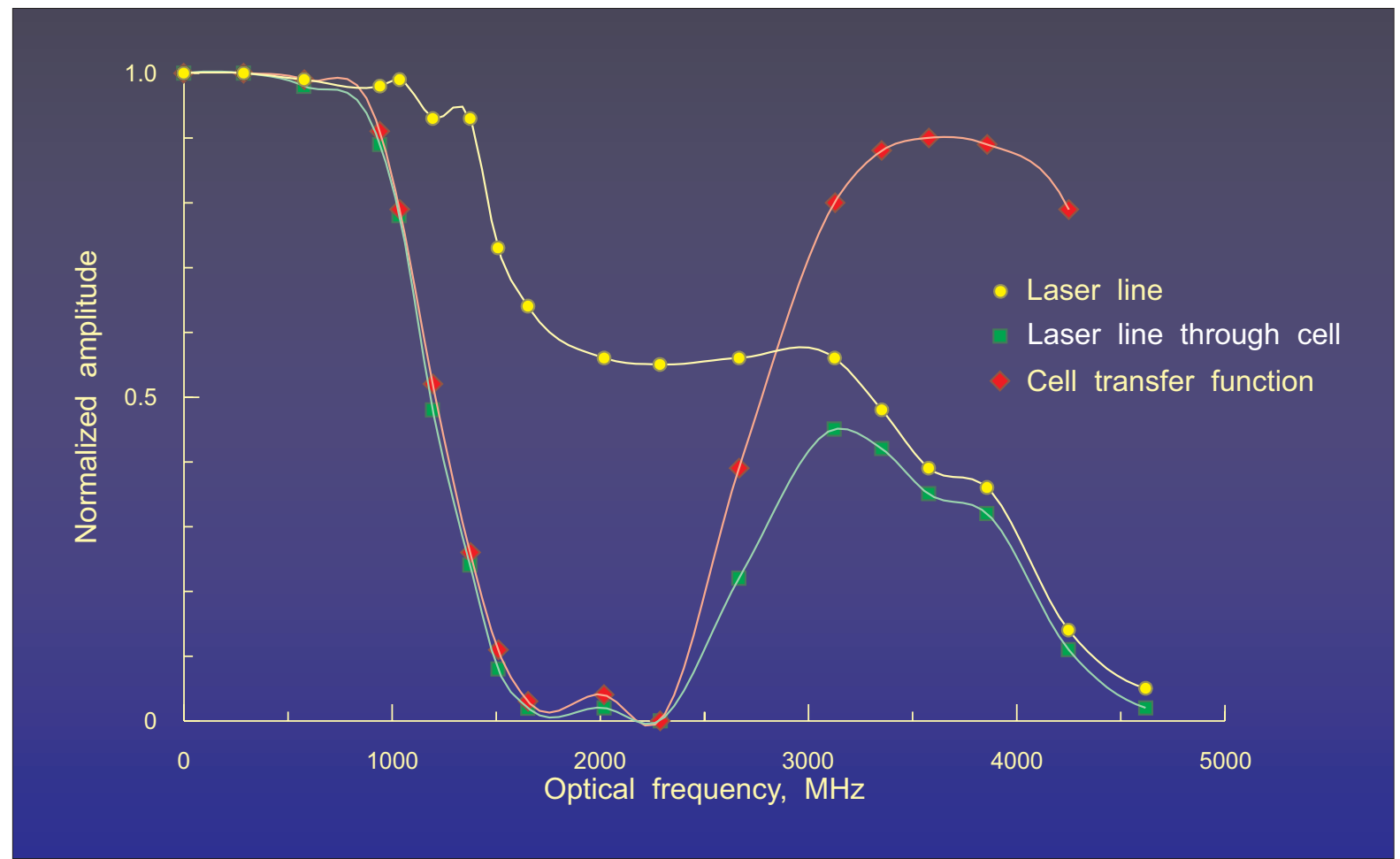

Figure $\quad$ - $\quad$ ransfer function of the odine absorption line filter,

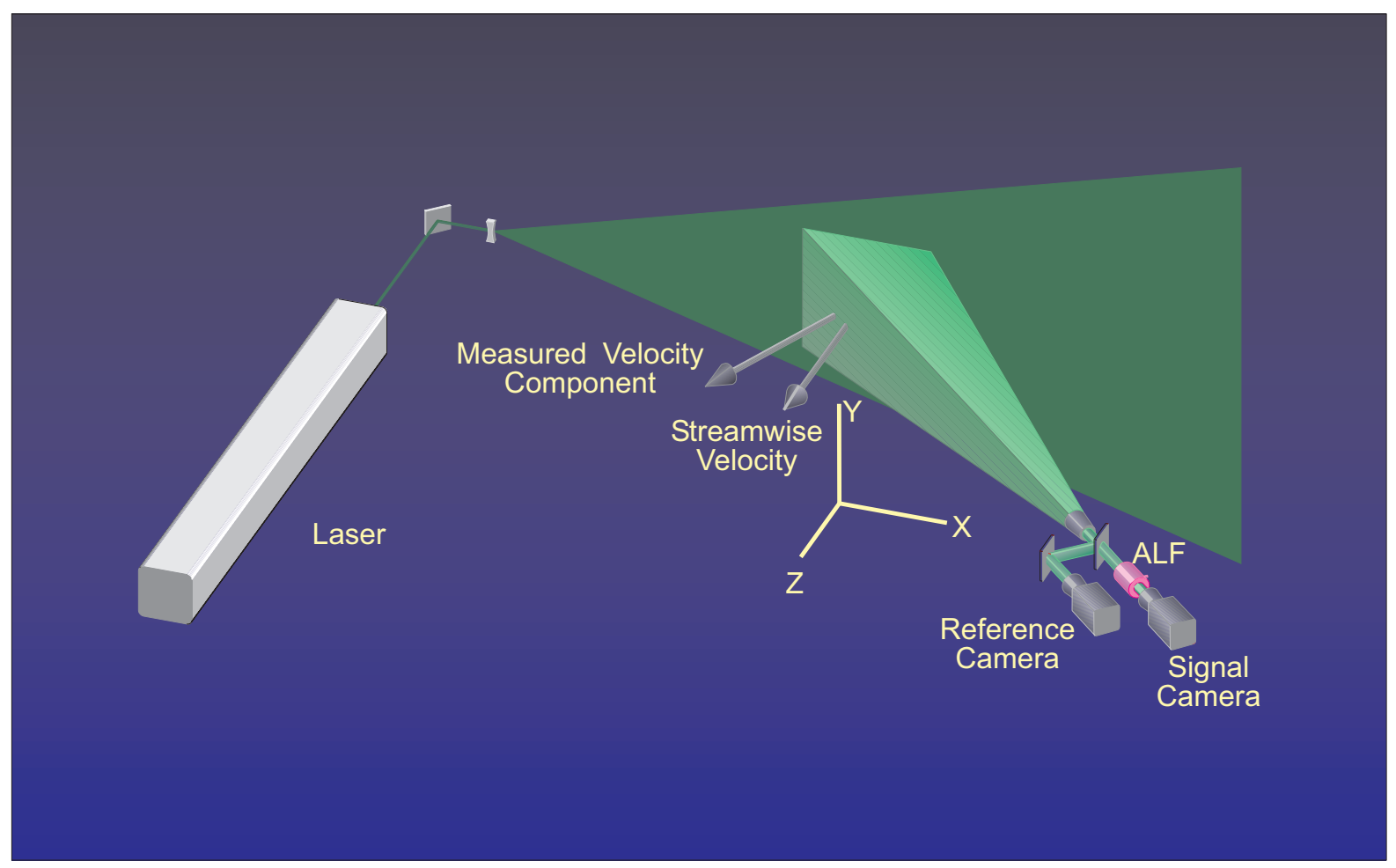

Figure

-

erodynamic raphical representation of the Doppler global velocimeter in the esearch unnel, 


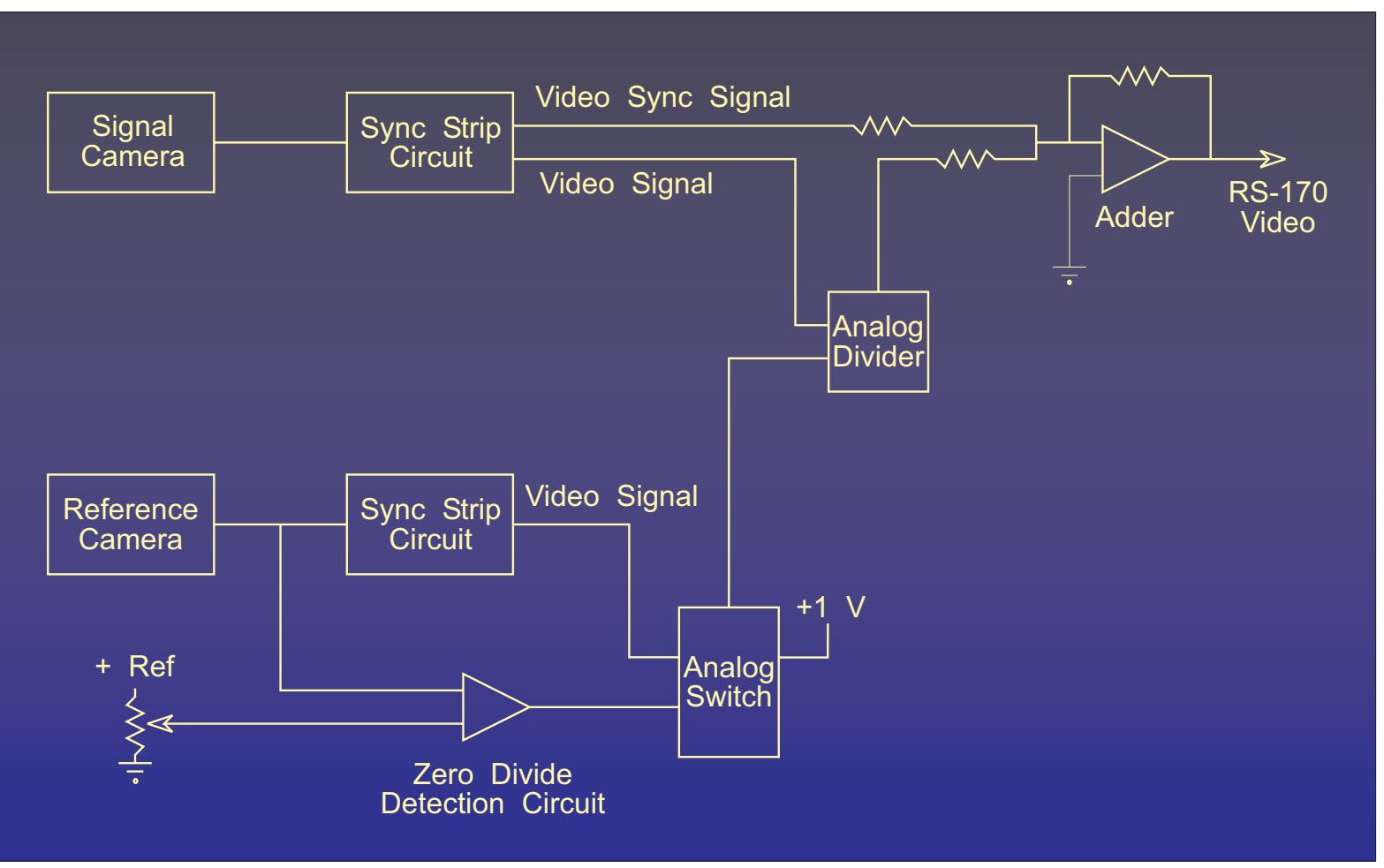
Figure
signal image by the reference image in real time.

ation ci

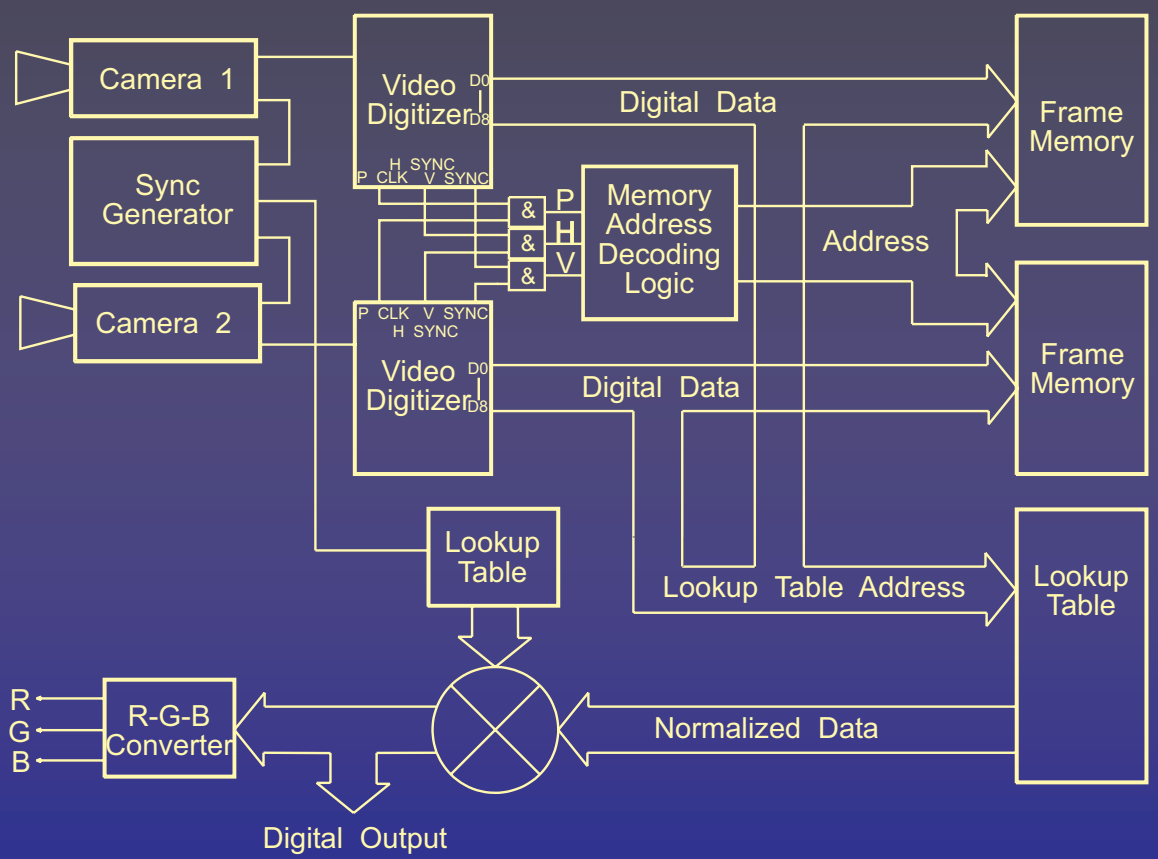

Figure

$10 \mathrm{c}$

diagram of the digital dual frame grabber for ac processing the images from the reference and signal cameras. 


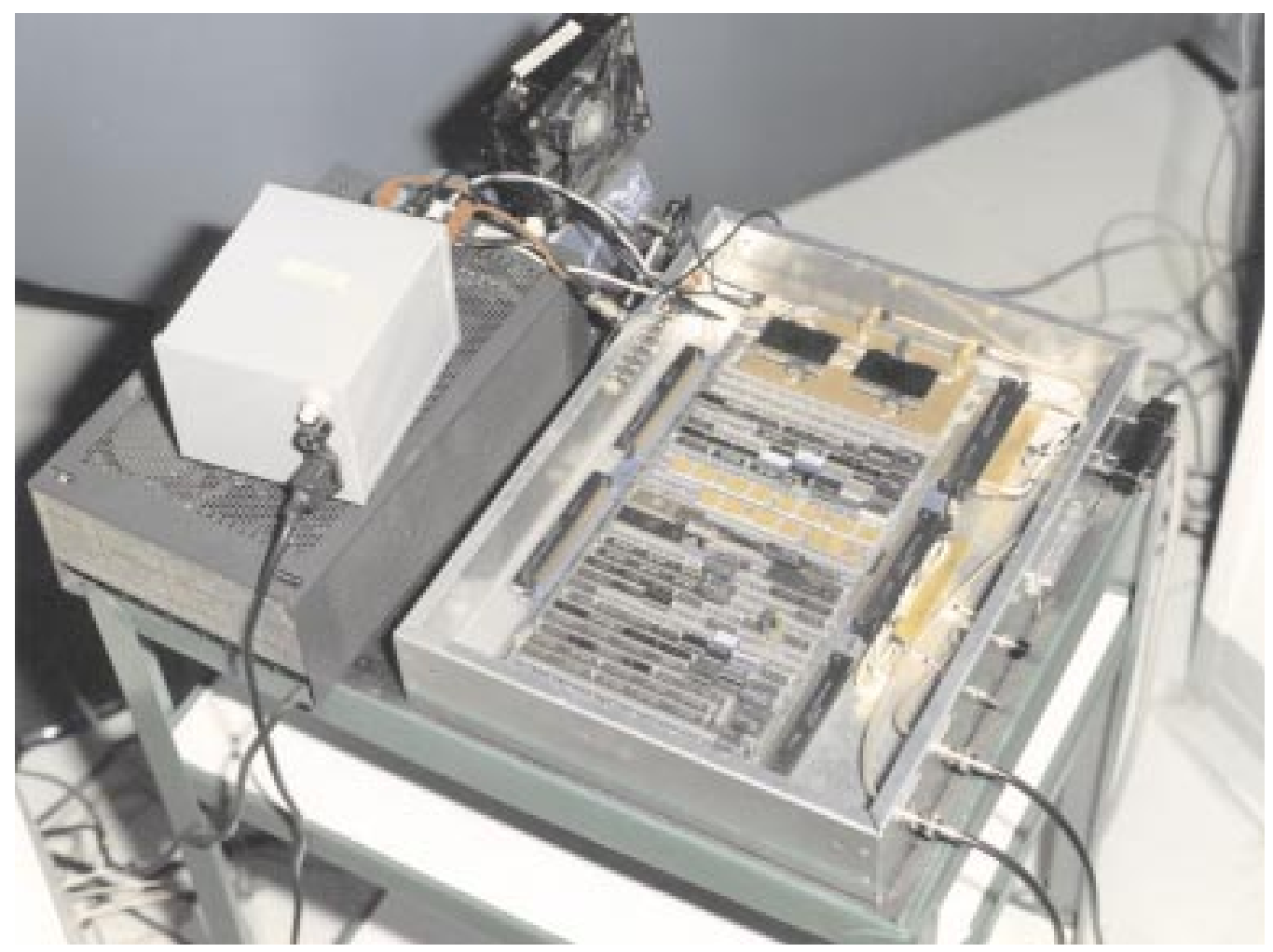

Figure

hotograph of the prototype digital dual frame grabber.

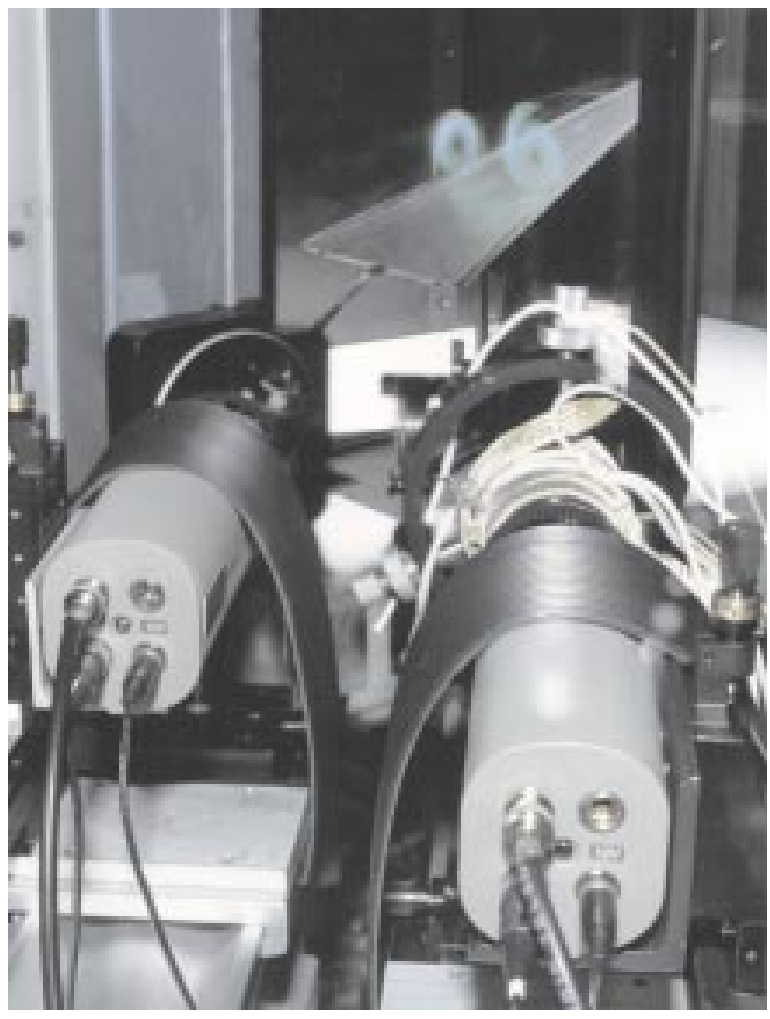

Figure iew of the laser light sheet above the delta wing from the receiver optics location. 


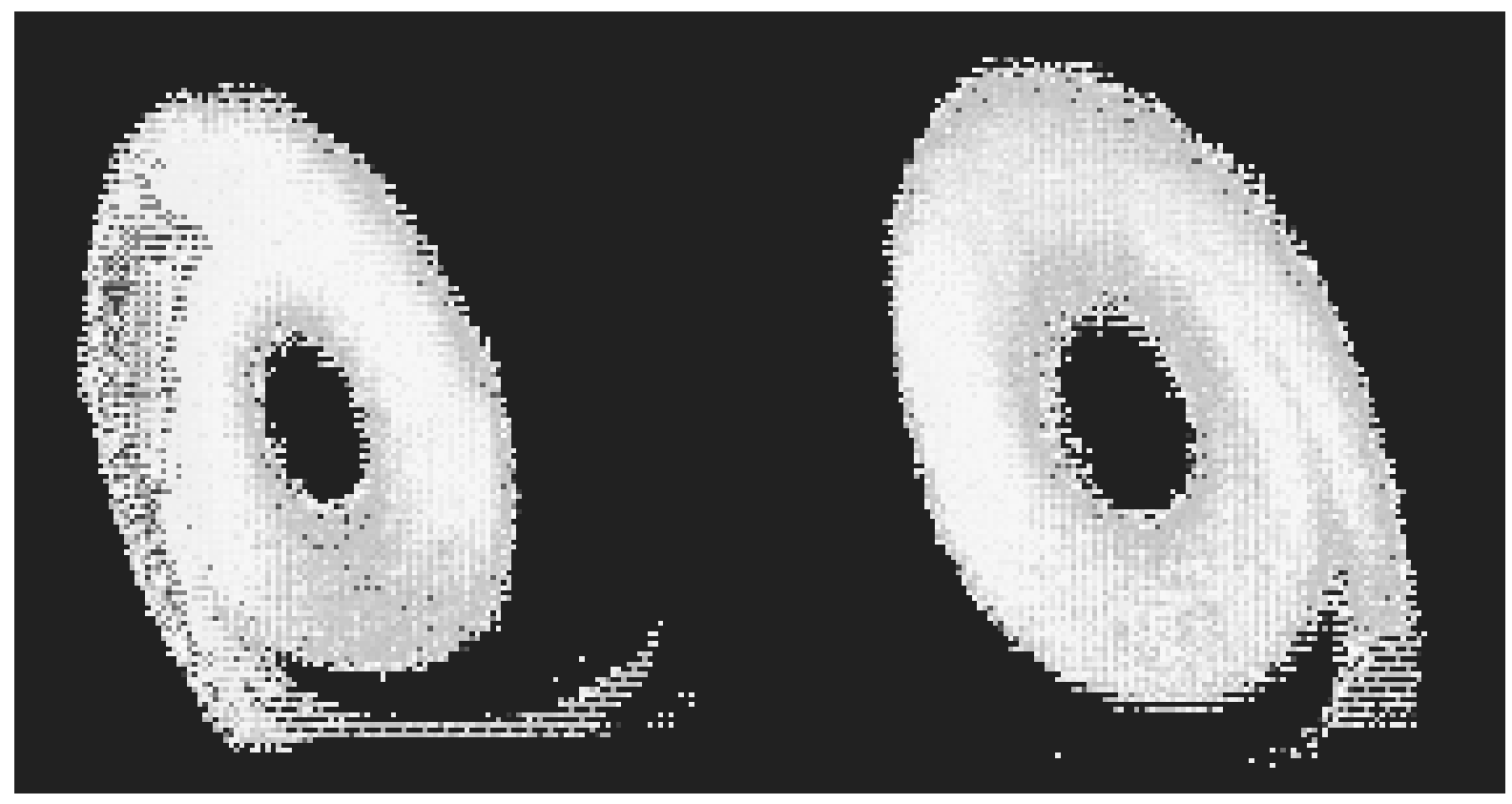

Figure .- ingle frame velocity contour map of the vorte delta wing at $2 \quad 0$ angle of attac streamwise direction in the hori , velocity componenfriosm 2 the ontal plane

-D measurements using digital signal processor.

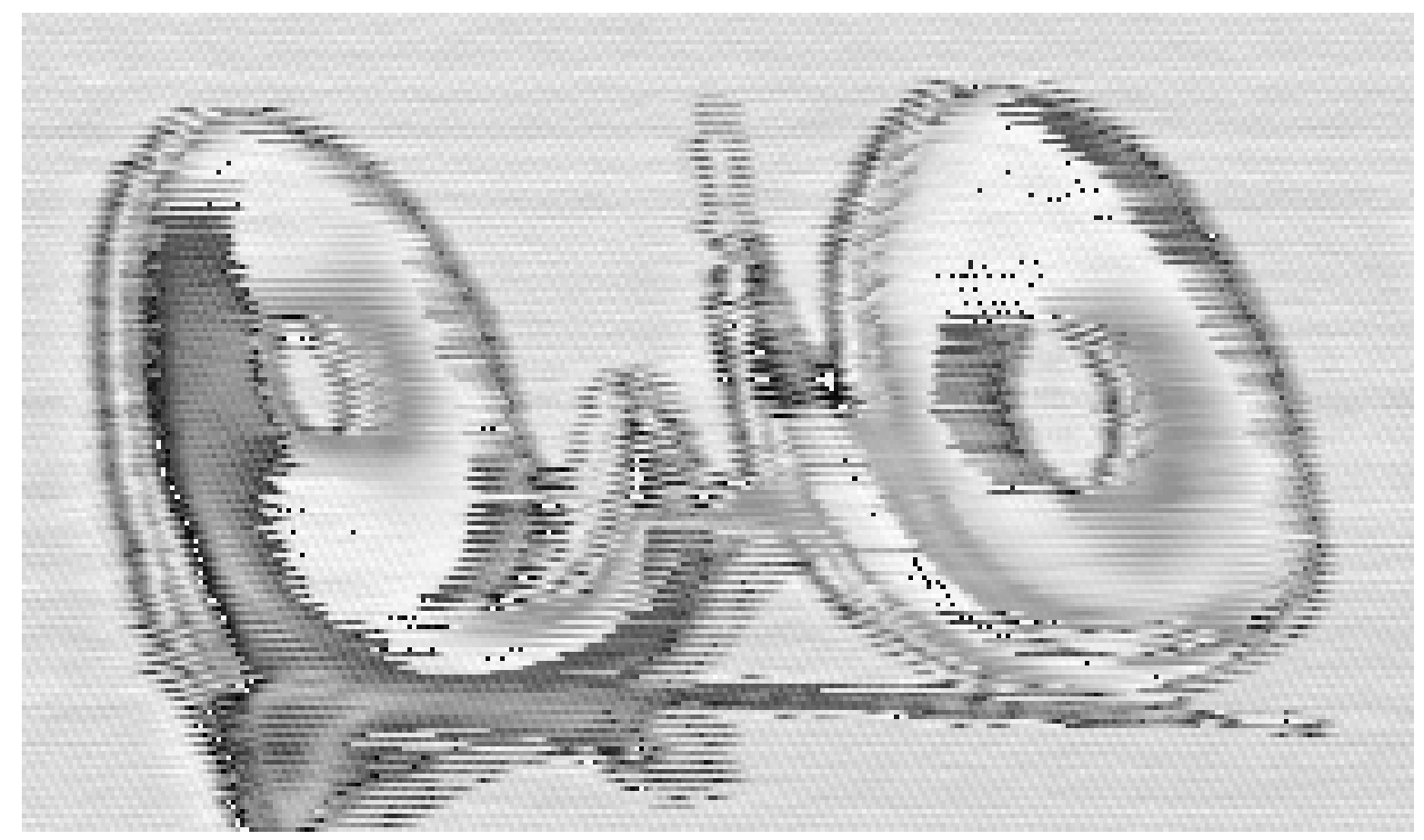

Figure 1 delta wing at $2{ }^{0}$ angle of attac streamwise direction in the hori

ingle frame velocity contour map of the vorte -D measurements using analog signal processor. 

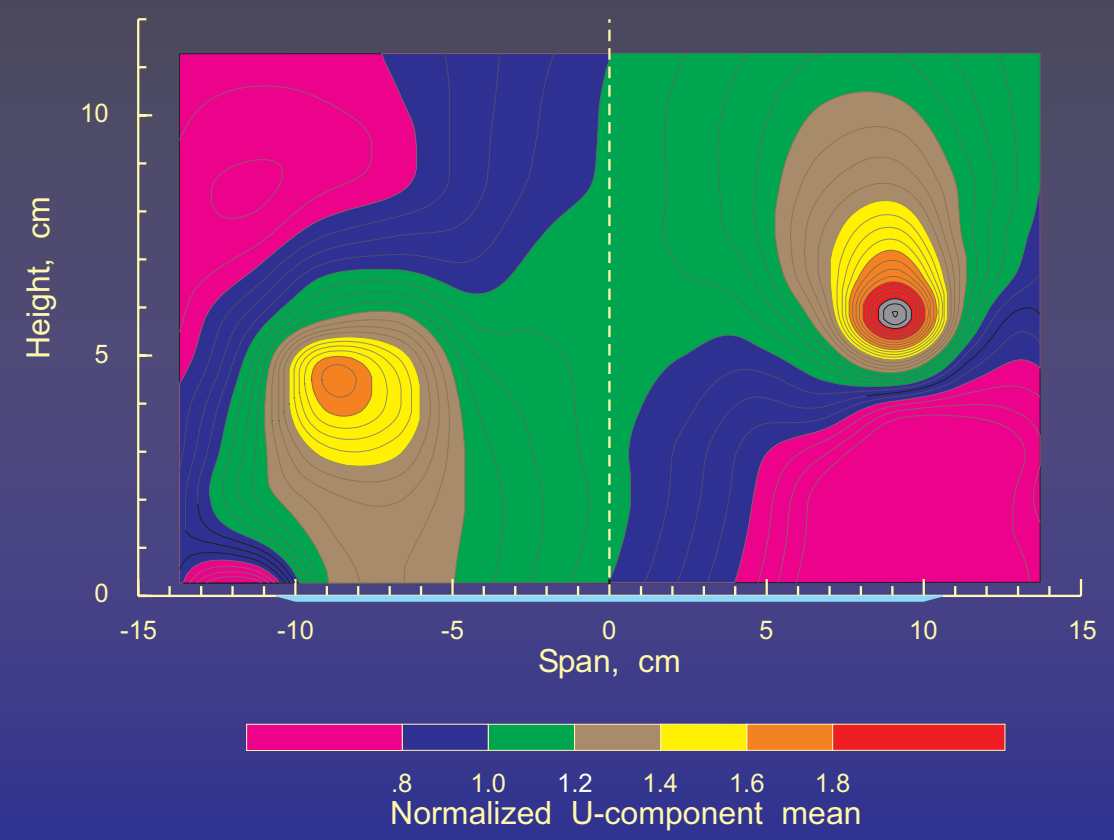

Figure 11.- elocity contour map of the vorte 2 angle of attac direction in the hori , velocity componenfriosm 2 the streamwise ontal plane -hole probe measurements.

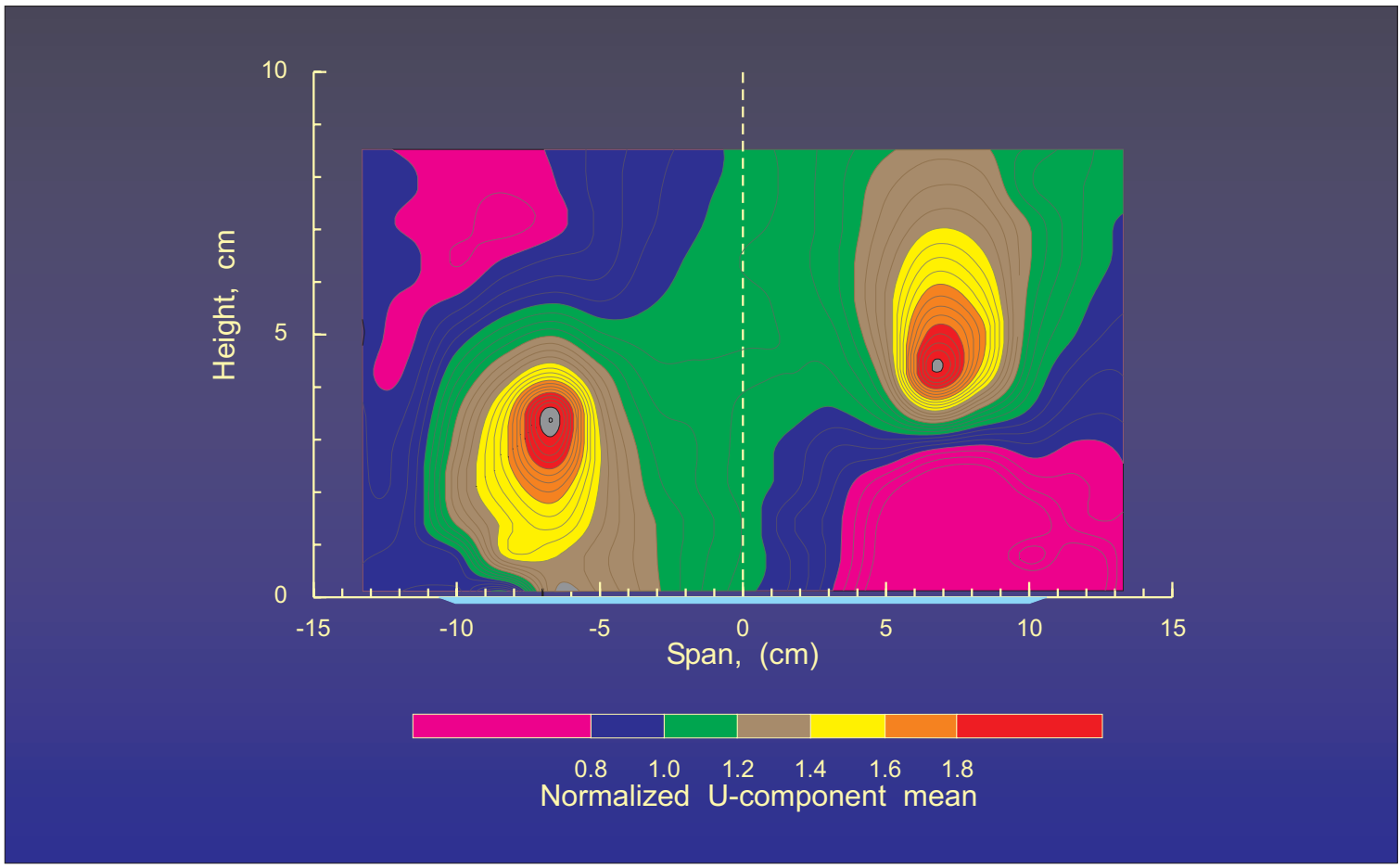

Figure 12.$2{ }^{0}$ angle of attac elocity contour map of the vorte flow field above a delta wing at direction in the hori , velocity componenfriosm 2 the streamwise o ntalla peaneelocimeter measurements. 


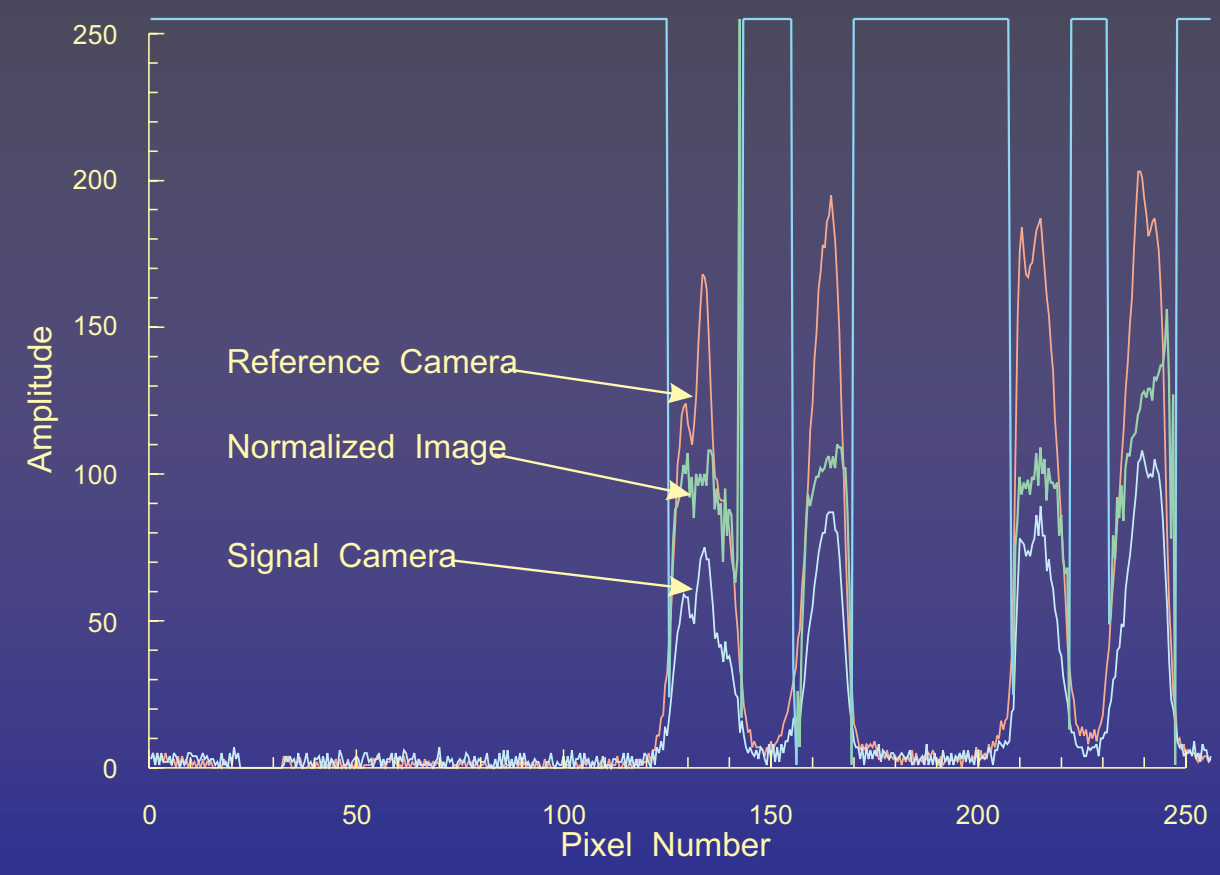

Figure 1

hori

ignal camera and reference camera signals and their ratio along ontal line passing through the center of the vortices, single frame.

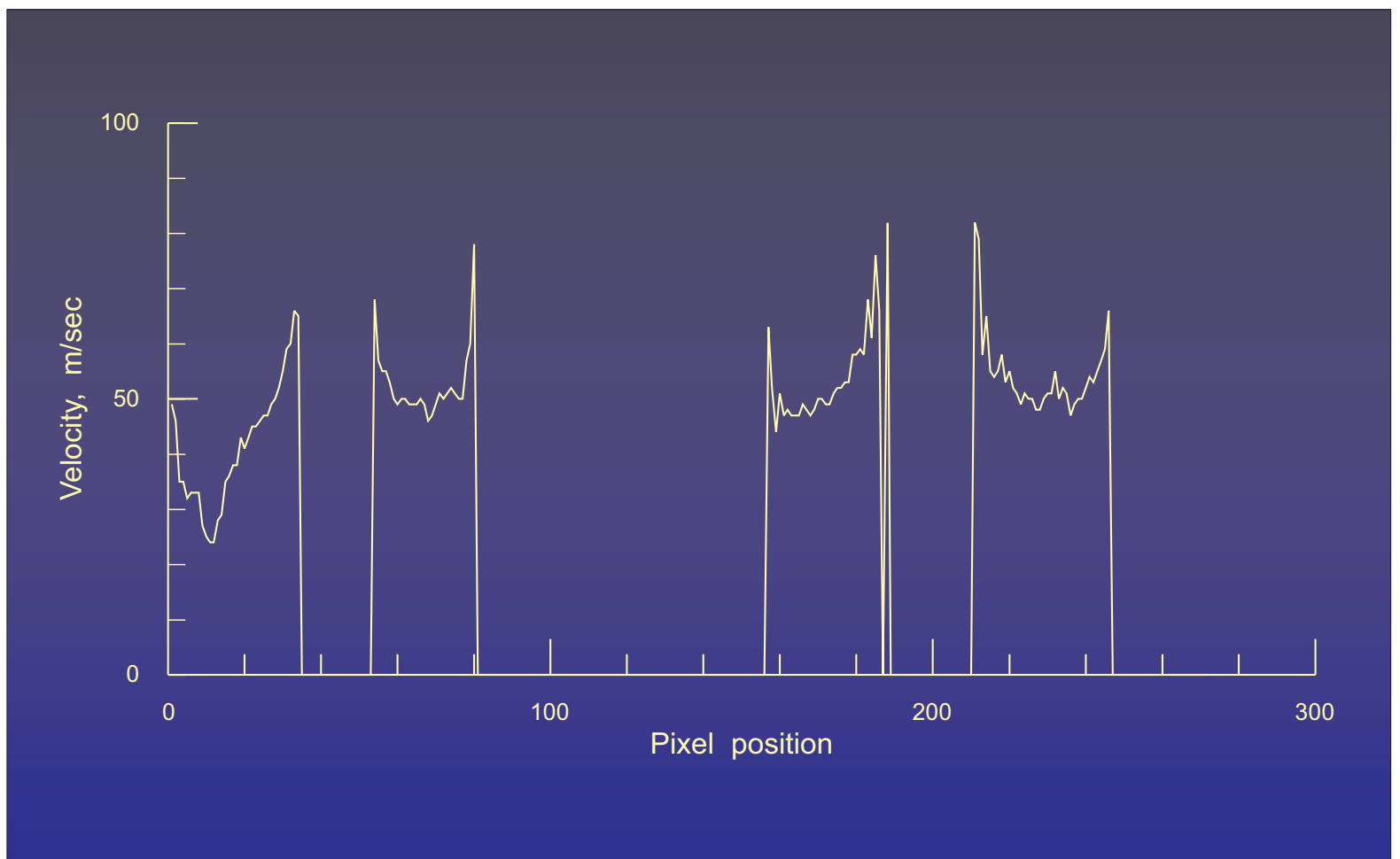

Figure 1

ormali the center of the vortices, average of 1

ed signal amplitude along a hori frames. 


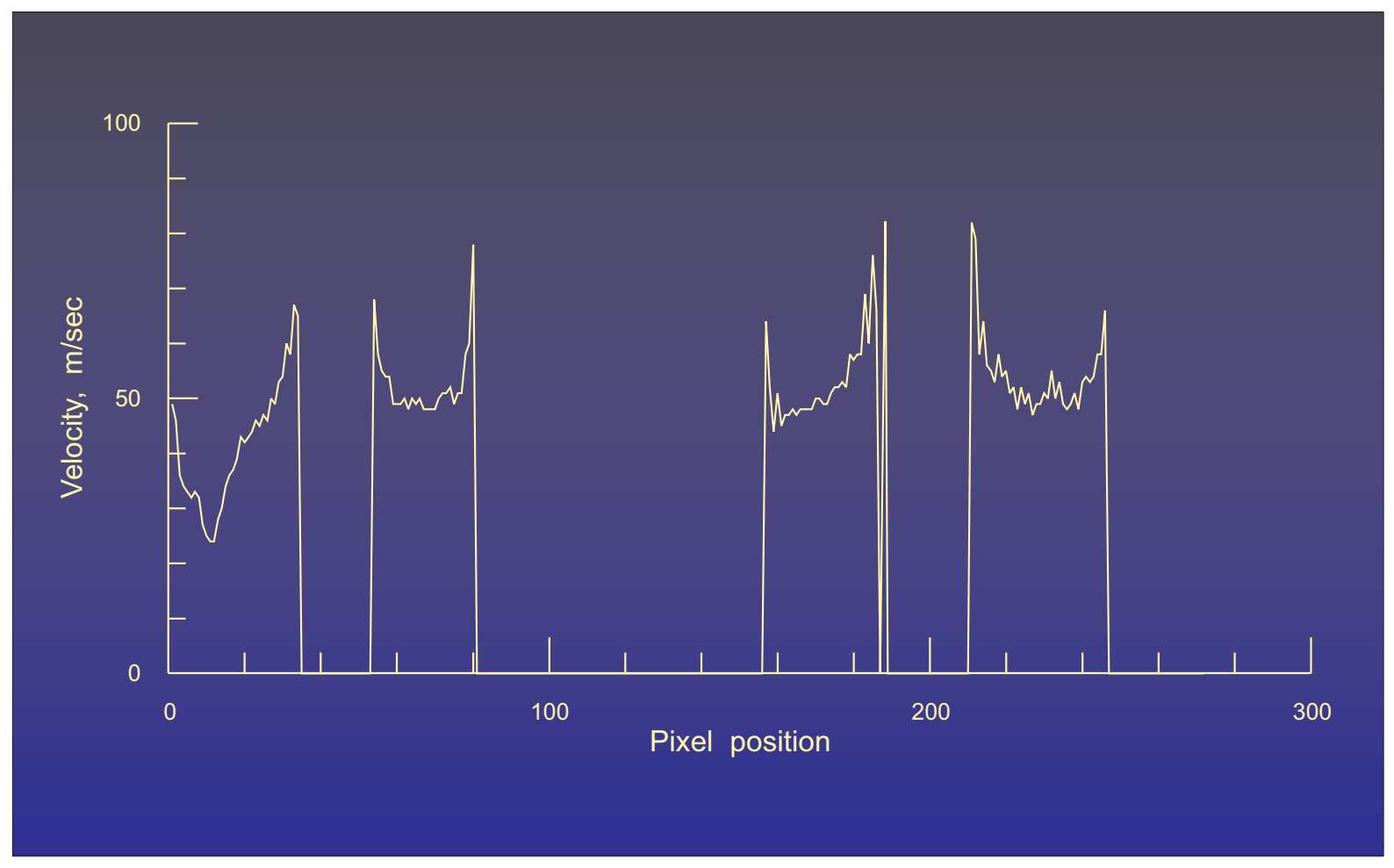
Figure 1
$\quad$ a hori 